\title{
On the production and survival of carbon fuel for superbursts on accreting neutron stars : Implications for mass donor evolution
}

\section{Citation}

Cooper, Randall L., Banibrata Mukhopadhyay, Danny Steeghs, and Ramesh Narayan. 2006. "On the Production and Survival of Carbon Fuel for Superbursts on Accreting Neutron Stars: Implications for Mass Donor Evolution." The Astrophysical Journal 642 (1): 443-54. https:// doi.org/10.1086/500922.

\section{Permanent link}

http://nrs.harvard.edu/urn-3:HUL.InstRepos:41384864

\section{Terms of Use}

This article was downloaded from Harvard University's DASH repository, and is made available under the terms and conditions applicable to Other Posted Material, as set forth at http:// nrs.harvard.edu/urn-3:HUL.InstRepos:dash.current.terms-of-use\#LAA

\section{Share Your Story}

The Harvard community has made this article openly available.

Please share how this access benefits you. Submit a story.

\section{Accessibility}


DRAFT VERSION FEBRUARY 5, 2008

Preprint typeset using $\mathrm{LT}_{\mathrm{E}} \mathrm{X}$ style emulateapj v. 6/22/04

\title{
ON THE PRODUCTION AND SURVIVAL OF CARBON FUEL FOR SUPERBURSTS ON ACCRETING NEUTRON STARS: IMPLICATIONS FOR MASS DONOR EVOLUTION
}

\author{
Randall L. CoOper, Banibrata Mukhopadhyay, Danny SteEghs, And Ramesh Narayan \\ Harvard-Smithsonian Center for Astrophysics, 60 Garden Street, Cambridge, MA 02138 \\ Draft version February 5, 2008
}

\begin{abstract}
We have investigated the physical conditions under which accreting neutron stars in low-mass X-ray binaries can both produce and preserve sufficient quantities of carbon fuel to trigger superbursts. Our theoretical models span the plausible ranges of neutron star thermal conductivities, core neutrino emission mechanisms, and areal radii, as well as the $\mathrm{CNO}$ abundances in the accreted material. We find that neutron stars that accrete hydrogenrich material with $\mathrm{CNO}$ mass fractions $Z_{\mathrm{CNO}} \lesssim Z_{\mathrm{CNO}, \odot}$ will not exhibit superbursts under any circumstances. Neutron stars that accrete material with $\mathrm{CNO}$ mass fractions $\gtrsim 4 Z_{\mathrm{CNO}, \odot}$ will exhibit superbursts at accretion rates in the observed range. On this basis, we suggest that the mass donors of superburst systems must have enhanced $\mathrm{CNO}$ abundances. The accreted $\mathrm{CNO}$ acts only as a catalyst for hydrogen burning via the hot $\mathrm{CNO}$ cycle, and therefore it is the sum of the three elements' mass fractions, not the individual mass fractions themselves, that is important. Systems that exhibit superbursts are observed to differ from those that do not exhibit superbursts in the nature of their helium-triggered Type I X-ray bursts: the bursts have shorter durations and much greater $\alpha$-values. Increasing the $\mathrm{CNO}$ abundance of the accreted material in our models reproduces both of these observations, thus once again suggesting enhanced CNO abundances in the mass donors. Many compact binary systems have been observed in which the abundances of the accreting material are distinctly non-solar. Though abundance analyses of the systems that exhibit superbursts currently do not exist, Bowen fluorescence blend profiles of $4 \mathrm{U}$ 1636-536 and Ser X-1 suggest that the mass donor stars may indeed have non-solar CNO metallicities. More detailed abundance analyses of the accreting matter in systems that exhibit superbursts are needed to verify our assertion that the matter is rich in CNO elements.

Subject headings: dense matter - nuclear reactions, nucleosynthesis, abundances - stars: neutron $-\mathrm{X}$-rays: binaries - X-rays: bursts
\end{abstract}

\section{INTRODUCTION}

Superbursts are energetic thermonuclear flashes that occur on the surfaces of accreting neutron stars in low mass $\mathrm{X}$-ray binaries. They are thought to be caused by unstable carbon burning deep within the accreted layer. Superbursts distinguish themselves from their hydrogen- and helium-burning Type I X-ray burst counterparts (which we refer to as "normal" bursts) by their $\sim 1000$ times larger burst energies and $\sim 1000$ times longer recurrence times (for reof this writing, nine superbursts have been observed in seven sources. Each of these superbursts has an integrated photon flux of $\approx 10^{42} \mathrm{ergs}$ and occurs in a system with an accretion rate $\dot{M} \approx 0.1-0.25 \dot{M}_{\text {Edd }}$, where $\dot{M}_{\text {Edd }}$ denotes the Eddington limit (Kuulkers 2004). Four superburst candidates have been observed in GX 17+2, which has an accretion rate $\dot{M} \approx 0.8 \dot{M}_{\text {Edd }}$ (in't Zand et al. 2004a,b). Very recently, Remillard et al. (2005a) and Kuulkers (2005) have observed two likely superbursts in the systems $4 \mathrm{U} 1608-522$ and $4 \mathrm{U}$ $0614+091$. The paucity of observational data makes the recurrence times of superbursts difficult to determine, though three superbursts have been observed within 4.7 years from the system 4U 1636-536 (Wijnands 2001; Strohmayer \& Markwardt 2002; Kuulkers et al. 2004).

Theoretical studies of superbursts have been rather successful at reproducing the general observational characteristics, such as the energetics, recurrence times, and absence of superbursts in systems with accretion rates below $\approx 0.1 \dot{M}_{\mathrm{Edd}}$ (Cumming \& Bildsten 2001; Strohmayer \& Brown 2002; views, see Cumming 2004; Strohmayer \& Bildsten 2005). As

Brown 2004; Cooper \& Narayan 2004, 2005; Cumming et al. 2005). Each of these models requires a carbon mass fraction $\gtrsim 0.1$ at the base of the accreted layer to trigger a thermonuclear instability. However, it is not understood why such a large amount of carbon should exist deep within the ocean. Previous theoretical calculations of both steady-state hydrogen/helium burning (Schatz et al. 1999, 2003) and unstable helium ignition (Schatz et al. 2001, 2003; Koike et al. 2004; Woosley et al. 2004; Fisker et al. 2005) on the surface of an accreting neutron star yield far too little carbon to ignite a superburst. Consequently, researchers who model superbursts must set the value of the carbon mass fraction at the base of the accreted layer "by hand," with little physical motivation. This is inadequate, since many superburst characteristics, such as the range of accretion rates in which superbursts occur (Cumming \& Bildsten 2001; Cooper \& Narayan 2005; Cumming et al. 2005) and the amount of energy released by neutrinos during a superburst (Cumming \& Bildsten 2001; Strohmayer \& Brown 2002; Cumming et al. 2005), are strong functions of the carbon mass fraction. Thus, no superburst model can be fully self-consistent until the process by which the carbon fuel survives both stable and unstable burning is understood.

As of this writing, each system in which a superburst has been observed exhibits normal Type I X-ray bursts as well (Kuulkers 2004; in't Zand et al. 2004a). The normal bursts these systems exhibit differ remarkably from the normal bursts of systems that accrete at similar rates and from which no superbursts have been observed. First, the average e-folding decay times are smaller than those of normal bursts

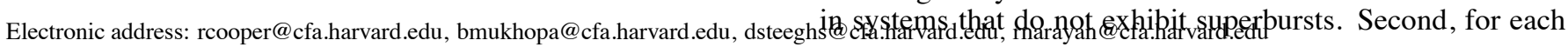


system that has exhibited a superburst, $\alpha \gtrsim 1000$, where $\alpha$ is defined as the ratio of the energy released between normal bursts to the energy released during a normal burst. This is significantly greater than the $\alpha$-values of most systems with similar accretion rates and in which no superbursts have been detected (Kuulkers et al. 2002b; in't Zand et al. 2003, 2004b). For systems with high accretion rates and in which the accreted matter is predominantly hydrogen, Narayan \& Heyl (2003) found that normal bursts occur in a unique regime that they refer to as "delayed mixed bursts." In these systems, a large fraction of the hydrogen and helium fuel burns stably before the full thermal instability is triggered. This stable burning explains the observations of van Paradijs et al. (1988a) and Cornelisse et al. (2003) who found that, for systems that accrete at a high rate and exhibit such Type I Xray bursts, the quantity $\alpha$ rises dramatically to values $\gtrsim 1000$. Cooper \& Narayan (2005) hypothesized that delayed mixed bursts may be the source of the substantial amount of carbon fuel needed to trigger a superburst in systems for which the accreted material is predominantly hydrogen. However, $\alpha$ values of the delayed bursts of Narayan \& Heyl (2003) reach $\sim 1000$ only if $\dot{M} \sim 0.3 \dot{M}_{\text {Edd }}$. For $\dot{M} \sim 0.1 \dot{M}_{\text {Edd }}$, roughly the lowest accretion rate at which delayed mixed bursts occur, they find $\alpha \lesssim 100$. The low $\alpha$-values for $\dot{M} \sim 0.1 \dot{M}_{\text {Edd }}$ are consistent with the results of previous and subsequent unstable helium ignition studies, all of which yield a negligible amount of carbon. As of yet, no model has reproduced the observed characteristics of normal bursts from systems that exhibit superbursts.

Any successful theoretical model of the low mass X-ray binaries considered in this paper must reproduce the following four phenomena that are unique to these systems. First, it must reproduce superbursts with energies and recurrence times that are consistent with observations. Second, nuclear burning in the oceans must produce sufficient amounts of carbon, and the carbon must survive until a superburst ignites. Third, delayed normal bursts with $\alpha \gtrsim 1000$ must occur. Fourth, the normal bursts that occur must have unusually short durations. Previous authors have made significant progress on the first phenomenon, but none has successfully addressed the latter three. In this investigation, we construct models that attempt to explain all four phenomena. We begin in $\$ 2$ with a description of our theoretical model. In $\$ 3$ we discuss the physical conditions under which sufficient amounts of carbon may survive long enough to ultimately fuel a superburst. We discuss the results of our model in $\$ 4$, and we compare our results to those of previous theoretical studies in $\$ 5$. In $\$ 6$ we compare our results with observations. Our model predicts that the matter accreted onto the surface of the neutron star must be rich in $\mathrm{CNO}$ in order for superbursts to occur. We discuss the observational evidence that such an overabundance exists in $\S 7$, and we conclude with a summary in $\S 8$.

\section{THE MODEL}

In this investigation, we assume that matter accretes spherically onto a neutron star of gravitational mass $M$ and areal radius $R$ at a rate $\dot{M}$, where $\dot{M}$ is the rest mass accreted per unit time as measured by an observer at infinity. We set the hydrogen and heavy element composition of the accreted matter to be that of the Sun, such that at the stellar surface the hydrogen mass fraction $X_{\text {out }}=0.7$ and the heavy element fraction $Z_{\text {out }}=0.004$, where $Z$ refers to all metals other than $C N O$. We set the helium mass fraction $Y_{\text {out }}=1-X_{\text {out }}-Z_{\text {out }}-Z_{\mathrm{CNO} \text {,out }}$, where $Z_{\mathrm{CNO} \text {,out }}$ is the mass fraction of $\mathrm{CNO}$ elements accreted from the companion star. We treat $Z_{\mathrm{CNO} \text {,out }}$ as a free parameter.

\subsection{Thermal and Hydrostatic Structure of the Crust}

To calculate the equilibrium configuration of the neutron star crust, we use the theoretical model of Cooper \& Narayan (2005) with two modifications. To calculate the inner temperature boundary condition at the crust-core interface, the authors assume that the core emits neutrinos via either modified Urca (mUrca) reactions or pionic reactions. They use the neutrino luminosities from Shapiro \& Teukolsky (1983) and thereby determine the core temperature. The core temperatures resulting from these neutrino luminosities are $\sim 3 \times 10^{8}$ $\mathrm{K}$ and $\sim 2 \times 10^{7} \mathrm{~K}$, respectively. However, if baryons exist in the core, they are probably superfluid at high densities (Baym et al. 1969). Baryon superfluidity greatly suppresses neutrino emission via mUrca reactions and therefore raises the core temperature (Yakovlev et al. 1999, 2001; Yakovlev \& Pethick 2004). Thus, when we model the effects of baryon superfluidity, we set the neutrino luminosity to be

$$
L_{\nu}=\left(2.0 \times 10^{37} \operatorname{ergs~s}^{-1}\right) \frac{m}{M_{\odot}}\left(\frac{T}{10^{9} \mathrm{~K}}\right)^{8},
$$

where $m$ is the interior gravitational mass and $T$ is the proper temperature, both evaluated at the crust-core interface. The core temperatures resulting from this neutrino luminosity are $\sim 6 \times 10^{8} \mathrm{~K}$. Note that we set the coefficient of $L_{\nu}$ not to correspond to any specific emissivity model, but rather to produce core temperatures in the desired range.

Cooper \& Narayan (2005) define the free parameter $C_{\mathrm{f}}$ as the fraction of hydrogen and helium that ultimately burns to carbon. This parameter enables the authors to set the value of $Z_{\mathrm{CNO} \text {,base }}$, the mass fraction of carbon at the base of the accreted layer, such that

$$
Z_{\mathrm{CNO}, \text { base }}=Z_{\mathrm{CNO}, \text { out }}+C_{\mathrm{f}}\left(X_{\text {out }}+Y_{\text {out }}\right) \text {. }
$$

The value of $C_{\mathrm{f}}$ should affect not only the carbon yield, but also the amount of energy generated within the crust, because the total energy released per unit mass when helium is burned ultimately to iron peak elements is greater than the energy released when helium is burned only to carbon. To account for the additional energy generation, we include $C_{\mathrm{f}}$ in the energy conservation equation. Thus, equation (6) of Cooper \& Narayan (2005) becomes

$$
\begin{array}{r}
e^{-2 \Phi / c^{2}} \frac{\partial}{\partial \Sigma}\left(\frac{F r^{2}}{R^{2}} e^{2 \Phi / c^{2}}\right)=T \frac{\mathrm{d} s}{\mathrm{~d} t}- \\
\left(\epsilon_{\mathrm{H}}+\left[1+\frac{E_{\mathrm{C}}^{*}}{E_{\mathrm{He}}^{*}}\left(1-C_{\mathrm{f}}\right)\right] \epsilon_{\mathrm{He}}+\epsilon_{\mathrm{C}}+\epsilon_{\mathrm{N}}-\epsilon_{\nu}\right) .
\end{array}
$$

\subsection{Nucleosynthesis in the Accreted Layer}

The thermal and hydrostatic structure model described above is quite sophisticated, approaching the state-of-the-art of such one-dimensional models. Its nuclear reaction network, however, is quite simplistic, since it includes reaction rates only for hydrogen, helium, and carbon burning. The hot nuclear flow through stable thermonuclear burning on the surface of an accreting neutron star almost certainly produces a wide assortment of different isotopes through hydrogen and triple- $\alpha$ reactions, rp- and $\alpha$ p-processes, and $\alpha$-captures. To 
calculate the detailed nuclear flow due to stable thermonuclear burning, we consider a large number of isotopes on either side of the stability line. Our nuclear reaction network contains 255 nuclear species up to ${ }^{72} \mathrm{Ge}$ and all of the possible reactions between the various isotopes. Stable burning at the sub-Eddington accretion rates we consider in this investigation produces negligible abundances of isotopes with atomic weights larger than 72 (e.g. Schatz et al. 1999), so the size of our network is adequate for our purposes. Lists of the major nuclear reactions that may take place in the flow are given in previous work (e.g. Clayton 1983; Lang 1999). In our nucleosynthesis code, we use the reaction rates of Fowler et al. (1975) but include the updated rates of Wagoner et al. (1967), Fuller et al. (1980, 1982a,b), Thielemann (1980), Wallace \& Woosley (1981), and Harris et al. (1983). Each of our reaction rates has been updated and is current up to the year 1996 or later (F.-K. Thielemann 1996, private communication). The code has been successfully used by one of us in previous studies of thermonuclear reactions in hot accretion discs (Chakrabarti \& Mukhopadhyay 1999; Mukhopadhyay \& Chakrabarti 2000, 2001). Therefore, we are confident that this code is applicable to our present investigation.

The nuclear reaction network, which is a set of coupled differential equations, is linearized and evolved in time along the thermal and hydrostatic structure of the ocean derived from the model described in $\$ 2.1$. This well-proven method is widely used in the literature (e.g. Arnett \& Truran 1969; Woosley et al. 1973; Mukhopadhyay \& Chakrabarti 2000). Here, we briefly outline how we time-evolve the isotopic abundances. For simplicity, we consider only four isotopes and only three reactions: ${ }^{1} \mathrm{H}\left(p, \beta^{+} \nu\right) \mathrm{D}, \mathrm{D}(\mathrm{D}, \gamma){ }^{4} \mathrm{He}$, and $2{ }^{4} \mathrm{He}(\alpha, \gamma){ }^{12} \mathrm{C}$, although, in our actual calculations, we use 255 isotopes and all of the possible reactions between the isotopes as mentioned above. Neglecting any backward reactions, the corresponding rate equations can be expressed as

$$
\frac{\mathrm{d}}{\mathrm{d} t}\left(\begin{array}{c}
X_{\mathrm{H}} \\
X_{\mathrm{D}} \\
X_{\mathrm{He}} \\
X_{\mathrm{C}}
\end{array}\right)=\left(\begin{array}{cccc}
-\lambda_{\mathrm{H}} & 0 & 0 & 0 \\
\lambda_{\mathrm{H}} & -\lambda_{\mathrm{D}} & 0 & 0 \\
0 & \lambda_{\mathrm{D}} & -\lambda_{\mathrm{He}} & 0 \\
0 & 0 & \lambda_{\mathrm{He}} & 0
\end{array}\right)\left(\begin{array}{c}
X_{\mathrm{H}} \\
X_{\mathrm{D}} \\
X_{\mathrm{He}} \\
X_{\mathrm{C}}
\end{array}\right)
$$

where the various $\lambda$ 's and $X$ 's are the reaction rates and mass fractions of the isotopes, respectively. The above equation can be written as

$$
\frac{\mathrm{d} \mathbf{v}}{\mathrm{d} t}=\Lambda \mathbf{v}
$$

If $\Lambda$ is diagnolizable, we can solve this equation by finding the four eigenvalues $\lambda_{i}$ and four eigenvectors $\mathbf{u}_{i}$ of $\Lambda . \mathbf{v}(t)$ is then a linear combination of the eigenvectors such that

$$
\mathbf{v}(t)=\sum_{i} A_{i} e^{\lambda_{i} t} \mathbf{u}_{i},
$$

where one determines the constant coefficients $A_{i}$ by setting $\mathbf{v}(0)=\mathbf{v}_{0}$, the initial elemental abundance. Note that this method is correct only if the individual nuclear lifetimes are constant. In our actual computational code, we include 255 isotopes, so $\Lambda$ is a $255 \times 255$ matrix with 255 eigenvalues and 255 eigenvectors, and thus $i$ runs from 1 to 255 .

To evolve the composition of the entire layer in time, we perform this procedure at each timestep. The reaction rates are in general functions of the temperature, density, and composition, and therefore they must be recalculated at each timestep. Normally, we set the timestep $\Delta t=\Delta \Sigma / \dot{\Sigma}$, where
$\Delta \Sigma$ is the thickness (mass per unit area) of a thin spherical shell and $\dot{\Sigma}$ is the mass accretion rate per unit area. However, we restrict the fractional change in the abundance of each species $i$ to be less than $\delta$, which we have set to 0.05 for our calculations. Specifically, for each species $i$ such that its mass fraction $X_{i} \geq 10^{-20}$, we require $\Delta t<\delta \min \left[X_{i} /\left(\mathrm{d} X_{i} / \mathrm{d} t\right)\right]$. Note that the fractional change in the abundance of each species with a nontrivial mass fraction is always much less than 0.05 . Nonetheless, we have performed calculations for values of $\delta$ both somewhat greater than and much less than 0.05 , and we have obtained the same results. Therefore, any error in our results due to our choice of stepsize is negligible compared to that due to the uncertainties in the reaction cross sections. We have verified that our nuclear reaction network conserves mass to within one part in $10^{8}$.

\subsection{Carbon Abundance in the Accreted Layer}

To calculate the mass fraction of carbon that exists at the base of the accreted layer, we proceed as follows. First, we calculate the equilibrium configuration of the neutron star crust as described in $\$ 2.1$. To perform this calculation, we choose an arbitrary value for the carbon mass fraction at the base of the layer. This value will be refined later. Second, we use this equilibrium configuration in our nucleosynthesis model to calculate the true carbon mass fraction at the base of the layer. In general, this value will be different than that used to determine the equilibrium configuration. Therefore, we repeat this two-step process, but we use the refined carbon mass fraction derived from our nucleosynthesis code to calculate the equilibrium configuration. We iterate this process until successive values of the carbon mass fraction determined from our nucleosynthesis model agree to sufficient accuracy. This process converges rapidly, and the value to which it converges is completely insensitive to the value of our initial guess. As an example of a typical convergence, the carbon mass fraction after each iteration for a particular calculation, starting with an initial guess of 0.2 , is 0.52709 , $0.56132,0.56477,0.56521$, and 0.56531 . After we have determined the carbon mass fraction, we conduct the full global linear stability analysis of Cooper \& Narayan (2005) to determine whether a superburst will occur, assuming that all of the carbon produced via stable burning has survived (see $\$ 3$ ).

The reader should note that we do not couple the nuclear energy generation rates of our reaction network directly into the energy conservation equation (3). In general, the contributions from hydrogen and helium burning dominate the total nuclear energy generation rate, and we calculate these contributions in equation (3) to high accuracy. Nevertheless, to ensure the internal consistency of our method, we have performed calculations in which the energy generation rates of our reaction network are coupled into equation (3), and we find that the differences in the final carbon mass fractions are insignificant.

\section{SURVIVAL OF THE CARBON FUEL}

Helium fusion via the triple- $\alpha$ reaction produces most of the carbon fuel that eventually triggers a superburst. Uncertainties in the carbon abundance deep within the accreted layer lie not in carbon's production, but in its survival. To ultimately become fuel for a superburst, carbon must survive both the stable burning that produces it and the unstable burning during normal Type I X-ray bursts that potentially consumes it.

\subsection{Survival During Stable Burning}


The rapid proton (rp) process of Wallace \& Woosley (1981) is the primary culprit in the destruction of ${ }^{12} \mathrm{C}$. If hydrogen is present, hydrogen will burn via the hot $\mathrm{CNO}$ cycle (Hoyle \& Fowler 1965). During the hot CNO cycle, essentially all of the CNO elements will be processed into ${ }^{14} \mathrm{O}$ and ${ }^{15} \mathrm{O}$. For the sub-Eddington accretion rates considered in this paper, the dominant breakout reaction from the hot CNO cycle into the rp-process is ${ }^{15} \mathrm{O}(\alpha, \gamma){ }^{19} \mathrm{Ne}$ (Wallace \& Woosley 1981; Schatz et al. 1999; Fisker et al. 2004). It is possible for ${ }^{19} \mathrm{Ne}$ to return to the hot CNO cycle by the series of reactions ${ }^{19} \mathrm{Ne}\left(\beta^{+} \nu\right){ }^{19} \mathrm{~F}(p, \alpha){ }^{16} \mathrm{O}(p, \gamma){ }^{17} \mathrm{~F}(p$, $\gamma)^{18} \mathrm{Ne}\left(\beta^{+} \nu\right){ }^{18} \mathrm{~F}(p, \alpha){ }^{15} \mathrm{O}$, but if ${ }^{19} \mathrm{Ne}$ captures a proton by the reaction ${ }^{19} \mathrm{Ne}(p, \gamma){ }^{20} \mathrm{Na}$, the ion can never return to the CNO cycle (Wallace \& Woosley 1981). Therefore, ${ }^{12} \mathrm{C}$ ion that is either accreted from the companion star or produced from the triple- $\alpha$ reaction will not survive if it is both converted to ${ }^{15} \mathrm{O}$ in the hot $\mathrm{CNO}$ cycle and removed from the hot $\mathrm{CNO}$ cycle via breakout reactions.

For the relatively high accretion luminosities considered in this investigation, the rate at which hydrogen burns is set by the $\beta$-decay timescales of ${ }^{14} \mathrm{O}$ and ${ }^{15} \mathrm{O}$, and therefore the rate is both temperature- and density-independent. The hydrogen nuclear energy generation rate for the hot CNO cycle is $\epsilon_{\mathrm{H}}=6 \times 10^{15} Z_{\mathrm{CNO}} \operatorname{ergs~g}^{-1} \mathrm{~s}^{-1}$ (Hoyle \& Fowler 1965). The column depth (mass per unit area) at which hydrogen burns out, $\Sigma_{\mathrm{H}}$, is thus

$$
\Sigma_{\mathrm{H}} \approx \dot{\Sigma} \frac{X_{\mathrm{out}} E_{\mathrm{H}}^{*}}{\epsilon_{\mathrm{H}}} \approx(\dot{\Sigma} \times 1100 \mathrm{~s}) \frac{X_{\mathrm{out}}}{Z_{\mathrm{CNO}, \text { out }}},
$$

where $\dot{\Sigma}$ is the mass accretion rate per unit area and $E_{\mathrm{H}}^{*}$ is the total nuclear energy released per unit mass of hydrogen burned. In general, the column depth at which hydrogen burns increases with increasing accretion rate.

In contrast, the rate at which helium burns is very sensitive to both temperature and density. The temperatures of the envelope and ocean of an accreting neutron star typically increase as the accretion rate increases. Consequently, for a pure column of helium, the column depth at which helium burns decreases with increasing accretion rate. This statement regarding the depth at which helium burns is not strictly true for a hydrogen/helium mixture because the hydrogen burning will steadily add helium to the matter, but the general idea is still valid.

If a large amount of carbon is to survive episodes of stable burning to eventually trigger a superburst, most of the accreted hydrogen must burn via the hot $\mathrm{CNO}$ cycle before helium starts to burn. If this occurs, then the carbon produced via helium burning will not be processed into ${ }^{15} \mathrm{O}$ and leave the $\mathrm{CNO}$ cycle through rp-process breakout reactions. Since the depth at which hydrogen burns increases with increasing accretion rate, and the depth at which helium burns decreases with increasing accretion rate, one would expect that more carbon will survive episodes of stable burning at lower accretion rates (Schatz et al. 1999, 2003). Figure 1 illustrates this trend.

\subsection{Survival During Unstable Burning}

Any carbon that survives stable burning must also withstand unstable burning in normal Type I bursts if the carbon is to ultimately become fuel for a superburst. Previous time-dependent models of normal prompt bursts, for which $\alpha \lesssim 100$, imply that essentially no carbon will survive unstable hydrogen and helium burning (Schatz et al. 2001,
2003; Koike et al. 2004; Woosley et al. 2004; Fisker et al. 2005). Cooper \& Narayan (2005) speculate that delayed bursts, which are bursts that are triggered after a long period of stable burning has occurred and thus result in extremely high $\alpha$-values, will leave most of the thick layer of carbon that exists below the ignition region intact. Presumably, the increase in temperature due to hydrogen and helium burning in the delayed burst is insufficient to ignite most of the carbon that exists deep in the ocean. Although we are currently unable to either confirm or refute this assertion, we note that observations do imply that a sufficient amount of carbon does indeed survive delayed bursts (in't Zand et al. 2003). Woosley et al. (2004) show that the ashes of a given normal burst are reprocessed in subsequent bursts. This "compositional inertia" may destroy some fraction of the carbon that a given burst leaves intact. However, Woosley et al. (2004) find that the effects of compositional inertia are diminished if the CNO abundance of the accreted material is high or if the burst recurrence time is long, both of which apply for the models we will suggest later in $§ 4$. Unfortunately, no detailed time-dependent studies of the delayed bursts observed in these systems have been carried out as of this writing. Therefore, our speculation that a large fraction of the carbon produced via stable burning will survive delayed bursts must be investigated further. For the purposes of this work, we assume that, if carbon is produced via stable burning, it will be destroyed if a normal prompt burst ignites (for which $\alpha \lesssim 100$ ), and it will survive if either a normal delayed burst ignites (for which $\alpha \gg 100$ ) or the system is stable to normal bursts.

\section{RESULTS}

We have constructed a total of 144 different models to determine the conditions under which accreting neutron stars both produce and preserve sufficient amounts of carbon to trigger superbursts. We choose four different stellar areal radii, three different core neutrino emission mechanisms, and three different conductive opacity prescriptions, which likely bracket the true radii, core neutrino emissivities, and conductive opacities of neutron stars found in nature. See Table 1 for a list of these parameters. The column "Core $\nu$ Emissivity" describes the neutrino emission mechanism in the core, where "hot mUrca" refers to a stellar core that cools via mUrca reactions suppressed by baryon superfluidity (see \$2.1), "mUrca", refers to a core that cools via mUrca reactions, and "Pion" refers to a core that cools via pionic reactions. The column " $Q$ " describes the conductive opacity of the crust. For this column, "5.2" and " 100 " refer to neutron stars with inner crusts that have formed ordered crystal lattices and which have impurity parameter values (Itoh \& Kohyama 1993; Brown 2000) of 5.2 and 100, respectively, and "disordered" refers to a neutron star with a completely disordered crust. See Brown (2004) and Cooper \& Narayan (2005) for details regarding these parameters and their effects upon superbursts characteristics. Additionally, we choose four different values of $Z_{\mathrm{CNO} \text { out }}$, the mass fraction of $\mathrm{CNO}$ elements accreted from the companion star.

A superburst will occur if a large amount of carbon deep within the ocean undergoes unstable thermonuclear fusion. In this investigation, we say that a superburst occurs if the carbon produced by stable helium burning survives (see §3.1) and the resulting carbon-rich accreted column is unstable according to the general-relativistic global linear stability analysis of Cooper \& Narayan (2005). For definiteness, we use the criterion that the carbon yield derived from our nucleosynthesis model will not survive if a normal burst occurs with $\alpha<500$. 

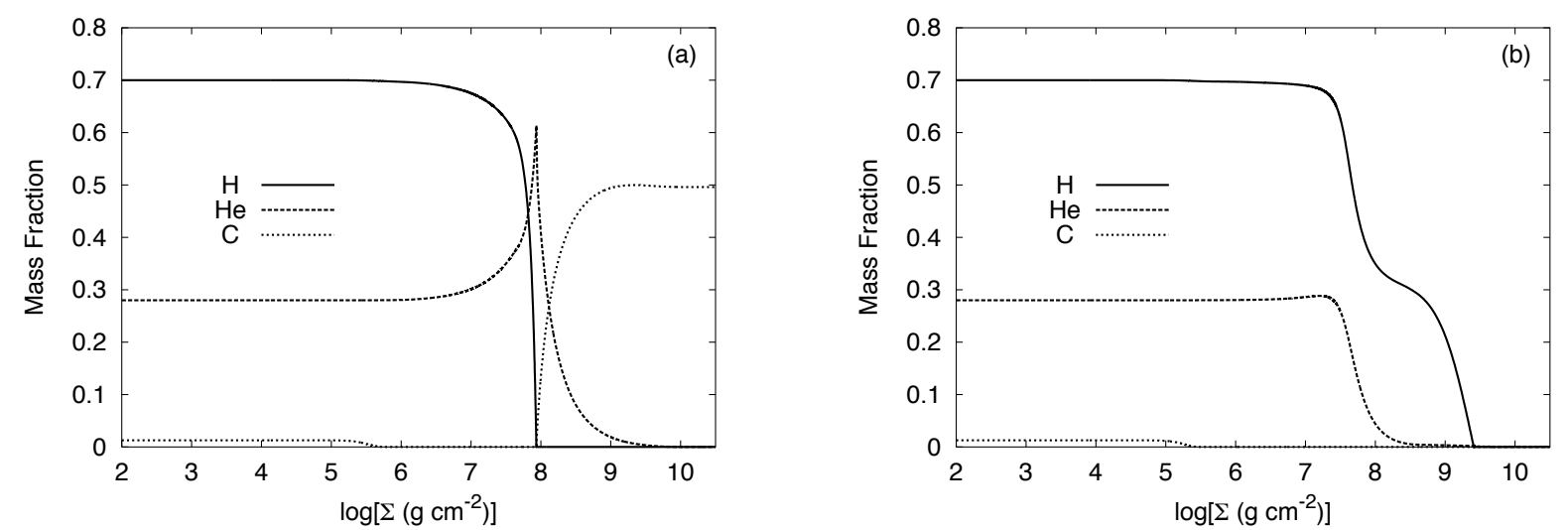

FIG. 1. $-{ }^{1} \mathrm{H},{ }^{4} \mathrm{He}$, and ${ }^{12} \mathrm{C}$ mass fractions in the accreted column due to stable burning for two neutron stars with different accretion rates. $\dot{M}=0.1 \dot{M}_{\text {Edd }}$ for Figure (a) and $\dot{M}=0.3 \dot{M}_{\text {Edd }}$ for Figure (b). Any ${ }^{12} \mathrm{C}$ accreted from the companion star is depleted when ${ }^{1} \mathrm{H}$ begins to burn because ${ }^{12} \mathrm{C}$ is processed into ${ }^{14} \mathrm{O}$ and ${ }^{15} \mathrm{O}$ during the hot $\mathrm{CNO}$ cycle. For $\dot{M}=0.1 \dot{M}_{\text {Edd }},{ }^{1} \mathrm{H}$ burns out before most of the ${ }^{4} \mathrm{He}$ has started to burn, so much of the ${ }^{12} \mathrm{C}$ produced via triple- $\alpha$ reactions survives. For $\dot{M}=0.3 \dot{M}_{\text {Edd }},{ }^{1} \mathrm{H}$ and ${ }^{4} \mathrm{He}$ burn simultaneously, so the ${ }^{12} \mathrm{C}$ produced via triple- $\alpha$ reactions is lost due to CNO cycle breakout reactions.

This cutoff value is close to that of the superbursting system with the smallest $\alpha$-value, $4 \mathrm{U} 1636-536$, for which $\alpha \approx 440$ (in't Zand et al. 2004b). The precise value of this cutoff is unimportant for the final results.

$$
\text { 4.1. Results for } Z_{\mathrm{CNO} \text {,out }}=Z_{\mathrm{CNO}, \odot}
$$

For the relatively high accretion rates at which superbursts occur, the envelope and ocean of the neutron star are thermally insulated from the inner crust and core, and so the thermal profile of the ocean depends primarily on the accretion rate (Cooper \& Narayan 2005). Consequently, the carbon yield resulting from stable hydrogen and helium burning is rather insensitive to both the conductive opacity of the crust and neutrino emission mechanism of the core, but it is quite sensitive to the accretion rate. We plot the ${ }^{12} \mathrm{C}$ mass fraction as a function of accretion rate for nine neutron stars with different conductive opacities and core neutrino emissivities in Figure 2a. The parameter $l_{\text {acc }}=\dot{M} / \dot{M}_{\text {Edd }}$ is the accretion rate normalized to the Eddington limit, where $\dot{M}_{\mathrm{Edd}}=4 \pi G M(1+z) / c z \kappa_{\mathrm{es}}$, $z=\left(1-2 G M / R c^{2}\right)^{-1 / 2}-1$ is the gravitational redshift, and $\kappa_{\mathrm{es}}=0.4 \mathrm{~cm}^{2} \mathrm{~g}^{-1}$. At lower accretion rates, most of the hydrogen burns before the helium ignites, so the carbon produced via stable helium burning survives. As the accretion rate increases, the hydrogen and helium ignition regions overlap, so the carbon produced via stable helium burning will be processed into oxygen during the hot $\mathrm{CNO}$ cycle, and the oxygen will be removed from the hot $\mathrm{CNO}$ cycle via breakout reactions (see \$3.1). Therefore, the carbon yield substantially decreases with increasing accretion rate.

Unlike the conductive opacity and core neutrino emissivity, the stellar radius significantly affects the carbon yield for a given $l_{\text {acc }}$, as illustrated in Figure $2 \mathrm{~b}$. There are two reasons for this. First, the accretion rate per unit area $\dot{\Sigma}$ is lower for a larger radius, so $\Sigma_{\mathrm{H}}$, the column depth at which hydrogen burns, decreases with increasing radius. Second, the density and temperature at a given column depth $\Sigma$ are generally lower for a larger radius, so the column depth at which helium burns increases with increasing radius. The combination of these two effects implies that helium is more likely to burn in a hydrogen-deficient medium if the stellar radius is large. Therefore, stable burning on neutron stars with larger radii will yield more carbon fuel.

The derived carbon yield for a given $l_{\text {acc }}$ shown in Figure
2 is meaningless, however, if prompt normal bursts occur at that $l_{\text {acc }}$. We plot the $\alpha$-values of normal bursts as a function of accretion rate for nine neutron stars with different conductive opacities and core neutrino emissivities in Figure $3 \mathrm{a}$ and with four different stellar areal radii in Figure 3b. To perform these normal burst calculations, we use the model described by Remillard et al. (2005b) tailored for persistent accretors, which is a slightly modified version of the model of Cooper \& Narayan (2005). Like the carbon yield due to stable burning, $\alpha$ depends weakly on the thermal structure of the crust and core, but it is quite sensitive to the stellar radius. For the $R=10.4 \mathrm{~km}$ models shown in Figure 3a, no normal bursts occur for $l_{\text {acc }} \gtrsim 0.3$. Delayed mixed bursts occur for $0.2 \lesssim l_{\text {acc }} \lesssim 0.3$, prompt bursts occur for $0.05 \lesssim l_{\text {acc }} \lesssim 0.2$, and delayed helium bursts occur for $l_{\text {acc }} \lesssim 0.05$ (for details on the various bursting regimes, see Narayan \& Heyl 2003).

We list the range of accretion rates in which superbursts occur for each model in Table 1. No superbursts occur at any accretion rate for any of the 36 models for which $Z_{\mathrm{CNO}, \text { out }}=Z_{\mathrm{CNO}, \odot \text {, where }} Z_{\mathrm{CNO}, \odot}=0.016$. For the high accretion rates at which either $\alpha \gg 100$ or normal bursts do not occur, stable burning produces a negligible amount of carbon, in agreement with Schatz et al. (1999, 2003). For $l_{\text {acc }} \sim 0.1$, prompt bursts destroy any carbon produced via stable burning (Schatz et al. 2001, 2003; Koike et al. 2004; Woosley et al. 2004; Fisker et al. 2005). At still lower $l_{\text {acc }}$, the carbon may survive the delayed bursts, but the carbon that remains will burn stably (Cumming \& Bildsten 2001; Cooper \& Narayan 2005; Cumming et al. 2005) and no superburst will occur. Increasing the radius does not raise the probability that a superburst will occur. Though increasing the radius will increase the carbon yield due to stable burning, it will also increase the limiting accretion rate above which delayed mixed bursts

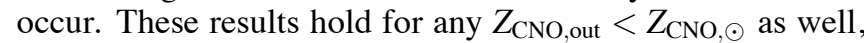
for decreasing $Z_{\mathrm{CNO} \text {,out }}$ will both lower the carbon yield and extend the range of accretion rates over which prompt normal bursts occur.

\subsection{Results for $Z_{\mathrm{CNO} \text { out }}>Z_{\mathrm{CNO}} \odot$}

As mentioned in $\$ 3.1$, carbon will survive to ultimately trigger a superburst only if the stable helium burning that produces it takes place in a hydrogen-deficient environment. Thus, the column depth at which the accreted hydrogen burns, 

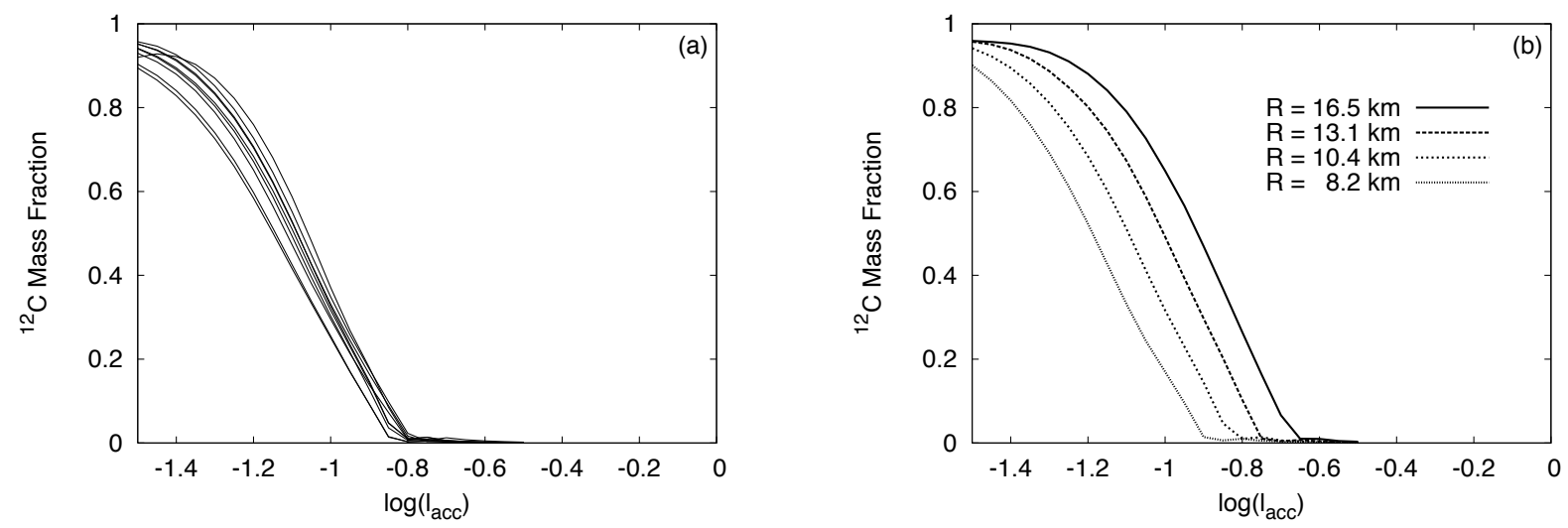

FIG. 2. $-{ }^{12} \mathrm{C}$ mass fraction at the the base of the accreted column due to stable burning as a function of accretion rate. The parameter $l_{\text {acc }}$ is the accretion rate normalized to the Eddington limit. Figure (a) shows the ${ }^{12} \mathrm{C}$ mass fractions deep within the accreted layer of $10.4 \mathrm{~km}$ neutron stars with $Z_{\mathrm{CNO} \text {,out }}=Z_{\mathrm{CNO}, \odot}$ for each of the nine thermal structure models. Figure (b) shows the ${ }^{12} \mathrm{C}$ mass fractions of neutron stars with $Z_{\mathrm{CNO} \text {,out }}=Z_{\mathrm{CNO}, \odot}, Q=5.2$ below the accreted layer, and with cores that emit neutrinos via mUrca reactions for four different radii.
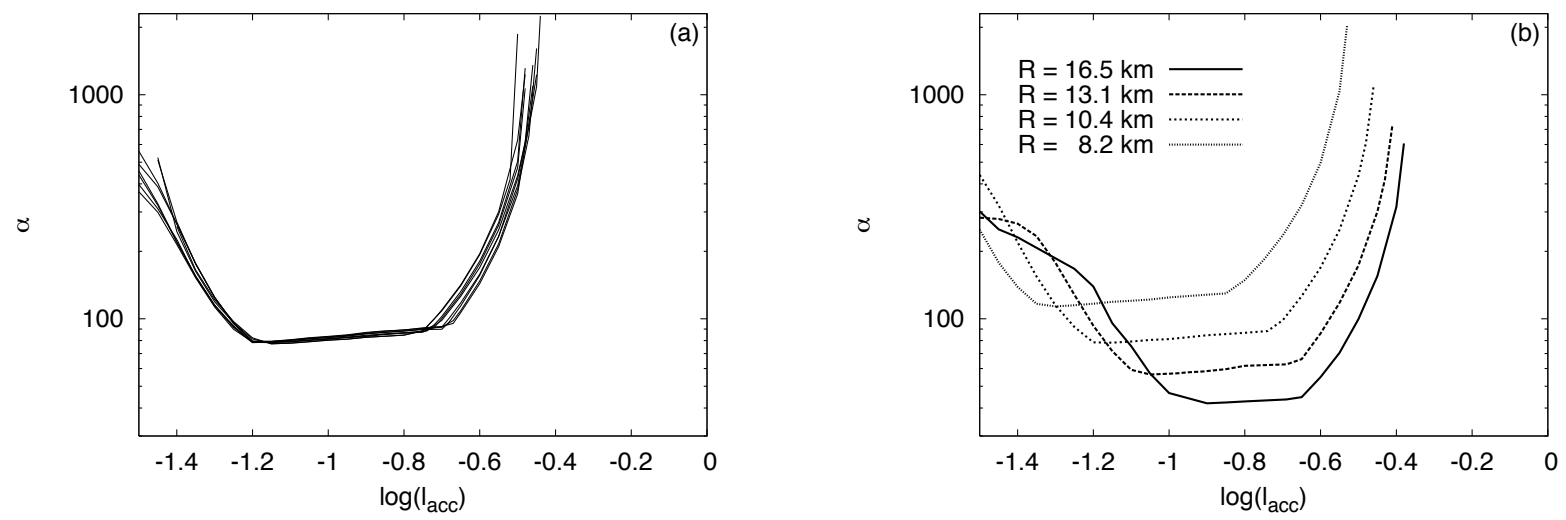

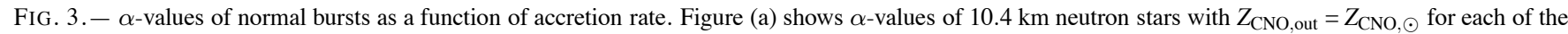
nine thermal structure models. Figure (b) shows $\alpha$-values of neutron stars with $Z_{\mathrm{CNO} \text {,out }}=Z_{\mathrm{CNO}, \odot}, Q=5.2$ below the accreted layer, and with cores that emit neutrinos via mUrca reactions for four different radii.

$\Sigma_{\mathrm{H}}$, must be less than the column depth at which helium burns to provide enough fuel for a superburst. According to equation (7), $\Sigma_{\mathrm{H}}$ is inversely proportional to $Z_{\mathrm{CNO} \text {,out }}$, so if the accreted matter is rich in CNO elements, hydrogen is more likely to burn out before helium ignites. Therefore, more of the carbon produced via stable helium burning should survive. Figure 4 illustrates this effect of the CNO mass fraction of the accreted matter on the carbon yield. We emphasize that the individual mass fractions of the accreted carbon, nitrogen, and oxygen are unimportant. Since the accreted CNO ions are simply catalysts for hydrogen burning, it is only the sum of the three individual mass fractions that matters.

As in $\S 4.1$, the derived carbon yield for a given $l_{\text {acc }}$ is meaningless if prompt normal bursts occur at that $l_{\mathrm{acc}}$. We plot the $\alpha$-values of normal bursts for four neutron stars with different CNO abundances of the accreted matter in Figure 5. Evidently, increasing $Z_{\mathrm{CNO} \text {,out }}$ affects normal bursts in several ways. First, it lowers the critical accretion rate above which normal bursts do not occur. Hydrogen burning is always $\beta$ limited at the accretion rates considered in this article, so only unstable helium burning triggers normal bursts. The helium nuclear energy generation rate $\epsilon_{\mathrm{He}}$ monotonically increases with temperature, but the temperature sensitivity decreases as temperature increases. In other words, $\partial^{2} \epsilon_{\mathrm{He}} / \partial T^{2}<0$ (Fushiki \& Lamb 1987). Therefore, helium burns stably at

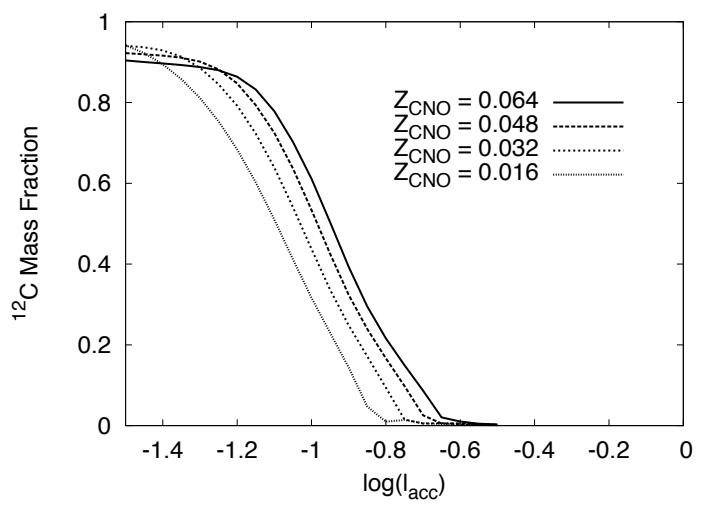

FIG. 4. $-{ }^{12} \mathrm{C}$ mass fraction at the the base of the accreted column due to stable burning as a function of accretion rate for four different values of $Z_{\mathrm{CNO} \text { out }}$, the mass fraction of $\mathrm{CNO}$ elements in the accreted matter. $R=10.4$ $\mathrm{km}, Q=5.2$ below the accreted layer, and the stellar core is assumed to emit neutrinos via mUrca reactions.

sufficiently high temperatures (Bildsten 1998). Since the temperature of the ocean increases with accretion rate, there exists a critical accretion rate above which normal bursts do not occur. The increased hydrogen nuclear energy generation rate due to the $\mathrm{CNO}$ enhancement raises the temperature of the 
TABLE 1

RESULTS

\begin{tabular}{|c|c|c|c|c|c|c|}
\hline \multirow[b]{2}{*}{$\begin{array}{c}R \\
(\mathrm{~km})\end{array}$} & \multirow[b]{2}{*}{$\begin{array}{c}\text { Core } \nu \\
\text { Emissivity }\end{array}$} & \multirow[b]{2}{*}{$Q$} & \multicolumn{4}{|c|}{$\dot{M} / \dot{M}_{\text {Edd }}$ Range for Superbursts for $Z_{\mathrm{CNO}, \text { out }}=$} \\
\hline & & & $Z_{\mathrm{CNO}, \odot}$ & $2 Z_{\mathrm{CNO}, \odot}$ & $3 Z_{\mathrm{CNO}, \odot}$ & $4 Z_{\mathrm{CNO}, \odot^{\mathrm{a}}}$ \\
\hline 8.2 & hot mUrca & 5.2 & & & & \\
\hline 8.2 & hot mUrca & 100 & & & $0.04-0.06$ & $0.04-0.08$ \\
\hline 8.2 & hot mUrca & disordered & & & $0.03-0.07$ & $0.03-0.10$ \\
\hline 8.2 & mUrca & 5.2 & & & $0.05-0.07$ & 0.03-0.09 \\
\hline 8.2 & mUrca & 100 & & & $0.03-0.07$ & $0.03-0.11$ \\
\hline 8.2 & mUrca & disordered & & & $0.03-0.07$ & $0.03-0.13$ \\
\hline 8.2 & Pion & 5.2 & & & $0.03-0.07$ & $0.03-0.06$ \\
\hline 8.2 & Pion & 100 & & & $0.03-0.07$ & $0.03-0.11$ \\
\hline 8.2 & Pion & disordered & & & $0.03-0.07$ & $0.03-0.14$ \\
\hline 10.4 & hot mUrca & 5.2 & & $0.04-0.05$ & $0.06-0.09$ & $0.07-0.13$ \\
\hline 10.4 & hot mUrca & 100 & & $0.03-0.05$ & $0.06-0.09$ & $0.07-0.13$ \\
\hline 10.4 & hot mUrca & disordered & & $0.03-0.05$ & $0.06-0.09$ & $0.07-0.13$ \\
\hline 10.4 & mUrca & 5.2 & & $0.03-0.05$ & $0.06-0.09$ & $0.07-0.13$ \\
\hline 10.4 & mUrca & 100 & & $0.03-0.05$ & $0.06-0.09$ & $0.07-0.13$ \\
\hline 10.4 & mUrca & disordered & & $0.03-0.05$ & $0.06-0.09$ & $0.07-0.13$ \\
\hline 10.4 & Pion & 5.2 & & $0.03-0.05$ & $0.06-0.09$ & $0.07-0.13$ \\
\hline 10.4 & Pion & 100 & & $0.03-0.05$ & $0.06-0.09$ & $0.07-0.13$ \\
\hline 10.4 & Pion & disordered & & $0.03-0.05$ & $0.06-0.09$ & $0.07-0.13$ \\
\hline 13.1 & hot mUrca & 5.2 & & & & $0.10-0.18,0.22$ \\
\hline 13.1 & hot mUrca & 100 & & & & $0.10-0.18,0.22$ \\
\hline 13.1 & hot mUrca & disordered & & & & $0.10-0.18,0.22$ \\
\hline 13.1 & mUrca & 5.2 & & & & $0.10-0.18,0.22-0.25$ \\
\hline 13.1 & mUrca & 100 & & & & $0.10-0.18,0.22$ \\
\hline 13.1 & mUrca & disordered & & & & $0.10-0.18,0.22$ \\
\hline 13.1 & Pion & 5.2 & & & & $0.10-0.18,0.22-0.25$ \\
\hline 13.1 & Pion & 100 & & & & $0.10-0.18,0.22-0.25$ \\
\hline 13.1 & Pion & disordered & & & & $0.10-0.18,0.22-0.25$ \\
\hline 16.5 & hot mUrca & 5.2 & & & & $0.04-0.07,0.14-0.32$ \\
\hline 16.5 & hot mUrca & 100 & & & & $0.04-0.07,0.14-0.32$ \\
\hline 16.5 & hot mUrca & disordered & & & & $0.04-0.07,0.14-0.32$ \\
\hline 16.5 & mUrca & 5.2 & & & & $0.04-0.07,0.14-0.32$ \\
\hline 16.5 & mUrca & 100 & & & & $0.04-0.07,0.14-0.32$ \\
\hline 16.5 & mUrca & disordered & & & & $0.04-0.07,0.14-0.32$ \\
\hline 16.5 & Pion & 5.2 & & & & $0.04-0.07,0.14-0.32$ \\
\hline 16.5 & Pion & 100 & & & & $0.04-0.07,0.14-0.32$ \\
\hline 16.5 & Pion & disordered & & & & $0.04-0.07,0.14-0.32$ \\
\hline
\end{tabular}

${ }^{\text {a}}$ For some models, superbursts occur over two distinct ranges of $\dot{M} / \dot{M}_{\text {Edd }}$ separated by a stable zone. Both $\dot{M} / \dot{M}_{\text {Edd }}$ ranges are given in these cases.

ocean, so helium is more likely to burn in a stable fashion, resulting in a lower critical accretion rate. Second, the $\alpha$-values of normal bursts are generally higher. Just below the critical accretion rate, the normal bursts that occur are "mixed" bursts, meaning that the bursts consume substantial amounts of both hydrogen and helium. Since $\Sigma_{\mathrm{H}}$ is lower, a larger fraction of the hydrogen burns before helium ignites unstably, which means that less hydrogen is consumed during the burst. This results in a higher $\alpha$. Third, the region of delayed helium bursts is curtailed, and a range of accretion rates exists below this region in which normal bursts do not occur. Again, this is due to the increased temperature of the ocean due to the intensified hydrogen energy generation rate.

We list the ranges of accretion rates at which superbursts

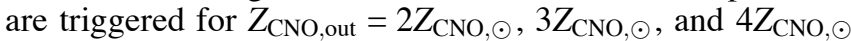
in Table 1. We find that an enhancement of the CNO abundance in the accreted matter will allow for superbursts to occur in certain ranges of accretion rates. The quantity $Z_{\mathrm{CNO}, o u t}$ is unique in that it is the only free parameter that will both increase the carbon yield due to stable burning at a given accretion rate and decrease the range of accretion rates at which prompt normal bursts occur. Therefore, we suggest that an

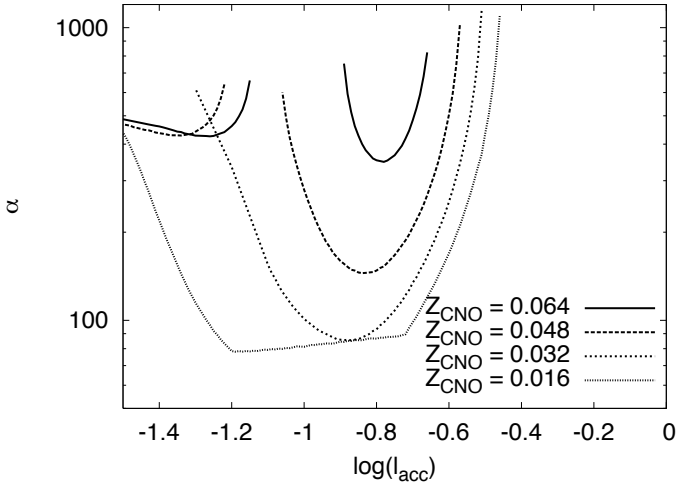

FIG. 5. $-\alpha$-values of normal bursts as a function of accretion rate for four different values of $Z_{\mathrm{CNO} \text {,out }} . R=10.4 \mathrm{~km}, Q=5.2$ below the accreted layer, and the stellar core is assumed to emit neutrinos via mUrca reactions.

enhancement of the $\mathrm{CNO}$ abundance in the accreted matter is a prerequisite for superbursts to occur in systems that accrete predominantly hydrogen. 


\section{COMPARISON WITH PREVIOUS THEORETICAL STUDIES}

As noted in $\$ 4.1$, our conclusion that neutron stars that

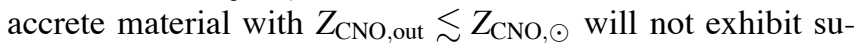
perbursts in consistent with the results of previous theoretical investigations. However, a comparison between Figure $2 \mathrm{a}$ of this work and Figure 2 of Schatz et al. (2003) illustrates that a discrepancy exists between the mass fraction of carbon that survives stable burning as a function of accretion rate that we calculate and the same quantity Schatz et al. (2003) derive. While both groups find that the carbon mass fraction $\approx 0.3$ at $l_{\text {acc }}=0.1$ (the lowest accretion rate they consider), Schatz et al. (2003) find that the mass fraction $\approx 0.08$ at $l_{\text {acc }}=0.3$, while our calculations imply that a negligible amount of carbon will survive at this accretion rate. Generally speaking, it appears that the resulting carbon mass fraction is more sensitive to the accretion rate in our model than it is in the model of Schatz et al. (2003). In particular, Figure $1 \mathrm{~b}$ illustrates that the hydrogen mass fraction plateaus around $\Sigma \approx 10^{8} \mathrm{~g} \mathrm{~cm}^{-2}$ in our model, whereas no such plateau exists in the model of Schatz et al. (2003). In this section we discuss our efforts to resolve this discrepancy.

One difference between the two models is that ours is general-relativistic, while theirs is Newtonian. Consequently, the gravitational acceleration in our model is greater. Although this will reduce the carbon yield somewhat, the effect is too small to account for such a large disparity. Furthermore, we would expect that the larger gravitational acceleration would reduce the carbon yield at all accretion rates, which means that the results of the two models should disagree at all accretion rates. Thus, we deduce that the inclusion of general relativity in our model is not the source of the discrepancy.

Another difference between the two models is the treatment of the inner boundary condition. While we set the inner boundary condition at the crust-core interface, Schatz et al. (2003) set it at the base of the burning layer. Therefore, we have conducted experimental calculations in which we set our boundary condition near the base of the burning layer. We are unable to reproduce their results at $l_{\mathrm{acc}}=0.3$ for any reasonable choice of boundary condition, and so we conclude that the treatment of the inner boundary condition is not the sole cause of the discrepancy.

A potential deficiency in our model may be that we do not directly couple our reaction network into our energy conservation equation. This could result in an inaccurate thermal profile which would affect our carbon yield. As discussed in $\$ 2.3$, we have carried out calculations, specifically at $l_{\text {acc }}=0.3$, in which the nuclear energy generation rate of our reaction network is directly coupled into equation (3), and the final carbon yield differs negligibly from our old result. Different radiative opacity prescriptions would affect the thermal profile too, but we use the same prescription as Schatz et al. (2003), so the opacity is likely not an issue either.

A plausible explanation for the discrepancy which we are not able to rule out is that some of the corresponding reactions rates in the two networks differ significantly. The two networks are of similar vintage, however, so we presume that none of the corresponding reaction rates disagrees considerably. Unfortunately, since we do not have access to the network of Schatz et al. (2003), we are unable to conduct a detailed comparison. However, two particular reactions that would certainly affect the final carbon yield and the hydrogen mass fraction profile are the $\mathrm{CNO}$ breakout re-

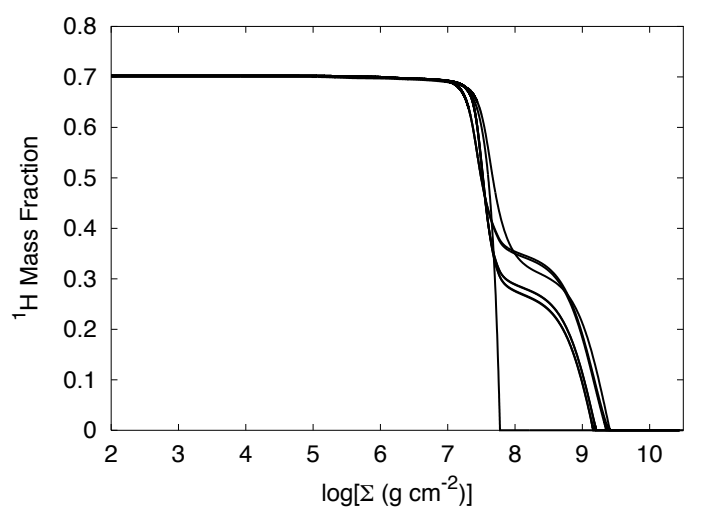

FIG. 6. $-{ }^{1} \mathrm{H}$ mass fraction profiles in the accreted column due to stable burning at $\dot{M}=0.3 \dot{M}_{\text {Edd }}$ for all possible combinations of the most recent ${ }^{15} \mathrm{O}(\alpha, \gamma){ }^{19} \mathrm{Ne}$ and ${ }^{14} \mathrm{O}(\alpha, \mathrm{p}){ }^{17} \mathrm{~F}$ reaction rates. The bottommost profile, where ${ }^{1} \mathrm{H}$ is depleted at $\Sigma \approx 10^{7.8} \mathrm{~g} \mathrm{~cm}^{-2}$, is the result of a calculation in which we artificially lowered both reaction rates by a factor of 10 and 100 , respectively.

actions ${ }^{15} \mathrm{O}(\alpha, \gamma){ }^{19} \mathrm{Ne}$ and ${ }^{14} \mathrm{O}(\alpha, \mathrm{p}){ }^{17} \mathrm{~F}$ (Schatz et al. 1999; Fisker et al. 2004). To investigate their effects, we repeated the calculation shown in Figure $1 \mathrm{~b}$ using all possible combinations of the three most recent ${ }^{15} \mathrm{O}(\alpha, \gamma){ }^{19} \mathrm{Ne}$ rates and the two most recent ${ }^{14} \mathrm{O}(\alpha, \mathrm{p}){ }^{17} \mathrm{~F}$ rates (J. L. Fisker 2005 , private communication). Figure 6 shows the resulting hydrogen mass fraction profiles. The $\mathrm{CNO}$ breakout reactions extract ${ }^{14} \mathrm{O}$ and ${ }^{15} \mathrm{O}$ from the $\mathrm{CNO}$ cycle, causing the hydrogen burning to stall. Hydrogen thus survives to a greater depth, which reduces the carbon yield. Although the lower ${ }^{15} \mathrm{O}(\alpha, \gamma){ }^{19} \mathrm{Ne}$ and ${ }^{14} \mathrm{O}(\alpha, \mathrm{p}){ }^{17} \mathrm{~F}$ reaction rates curtail the plateau somewhat, we find that none of the six possible reaction rate combinations eliminates the plateau entirely. Furthermore, all of the final carbon yields differ by less than a factor of 2 . We are able to remove the hydrogen plateau only by artificially reducing the ${ }^{15} \mathrm{O}(\alpha, \gamma){ }^{19} \mathrm{Ne}$ and ${ }^{14} \mathrm{O}(\alpha, \mathrm{p}){ }^{17} \mathrm{~F}$ reaction rates by a factor of 10 and 100 , respectively. When we do this, we find that the carbon mass fraction $\approx 0.08$ for $l_{\text {acc }}=0.3$.

In summary, we are unable to pinpoint the reason why the final carbon yield due to stable burning at relatively high accretion rates derived from our model differs from the yield derived by Schatz et al. (2003). Fortunately, our general conclusions remain unchanged regardless of which model is a better description of the nuclear reactions that occur in nature, for even if the model of Schatz et al. (2003) is "correct," we still find that the mass donors in systems that exhibit superbursts must be evolved stars with enhanced CNO abundances in order to both generate sufficient amounts of carbon fuel and reproduce the observed characteristics of the normal bursts.

\section{COMPARISON WITH OBSERVATIONS}

The nine superbursts that have been observed as of this writing, excluding those from the anomalous system GX $17+2$, occurred in systems with accretion rates between $10 \%$ and $25 \%$ of the Eddington limit. The normal bursts that these systems exhibit differ in two ways from the normal bursts in other systems with similar accretion rates. First, the normal bursts are delayed bursts, with $\alpha \gtrsim 1000$ (Kuulkers et al. 2002a; in't Zand et al. 2003, 2004b), whereas most systems accreting at these rates exhibit prompt bursts, with $\alpha<100$ (van Paradijs et al. 1988a; Narayan \& Heyl 2003). Second, the average durations of normal bursts that occur in these 
systems are shorter than those of normal bursts in systems that do not exhibit superbursts (in't Zand et al. 2003, 2004b). Any successful theoretical model of superbursts must be able to explain these facts. In this investigation, we concentrate on systems in which the accreted matter is predominantly hydrogen. This excludes the system 4U 1820-303, in which the accreted matter is probably dominated by helium (Strohmayer \& Brown 2002; Cumming 2003). It is likely that the long periods of stable helium burning that take place in the hydrogen-deficient ocean of this source produce large amounts of carbon fuel for superbursts. We note, however, that it is not understood theoretically why this system does not exhibit normal bursts during these periods, when the accretion rate is near its maximum (Bildsten 1995, 1998; Cumming 2003).

We begin by discussing the range of accretion rates in which superbursts have been observed, $l_{\text {acc }} \approx 0.1-0.25$. From Table 1 , we require that $Z_{\mathrm{CNO} \text {,out }} \gtrsim 4 Z_{\mathrm{CNO}, \odot}$ in order for a superburst to occur in this range. $Z_{\mathrm{CNO} \text { out }}$ is the most important parameter that determines whether a system in this range of accretion rates will exhibit a superburst. If the $\mathrm{CNO}$ mass fraction of the accreted matter is low, we find that there is no scenario in which a sufficient amount of carbon fuel will survive to ultimately trigger a superburst. The second most important parameter is the stellar areal radius. Although a neutron star with almost any plausible radius can exhibit a superburst at $l_{\text {acc }} \approx 0.1$ given a sufficiently large value of $Z_{\mathrm{CNO} \text {,out }}$, neutron stars will not exhibit superbursts at $l_{\text {acc }} \approx 0.2$ unless the radius $R \gtrsim 13 \mathrm{~km}$. If all neutron stars are indeed rather large, then our model predicts that superbursts will not occur in systems that accrete predominantly hydrogen if $l_{\text {acc }}<0.1$, in agreement with observations. Our model may also explain why superbursts are not observed in most low mass X-ray binaries with $l_{\text {acc }} \gtrsim 0.3$ (excluding the anomalous GX $17+2$, which we will discuss below).

Previous theoretical models of normal bursts all produce prompt bursts at $l_{\text {acc }} \approx 0.1$, with $\alpha \lesssim 100$. The results of these models are inconsistent with the delayed bursts observed at $l_{\text {acc }} \approx 0.1$ from the systems $4 \mathrm{U} 1636-536, \mathrm{KS} 1731-260$, and 4U 1254-690, in which $\alpha \approx 440,780$, and 4800, respectively (in't Zand et al. 2003, 2004b). These models usually as-

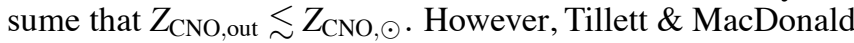
(1992) showed that theoretical models of normal bursts from the superburster 4U 1636-536 are inconsistent with observations unless a significant amount of $\mathrm{CNO}$ exists in the ocean. Figure 5 shows that an enhancement of the $\mathrm{CNO}$ abundance in the accreted matter produces delayed bursts at $l_{\text {acc }} \approx 0.1$ that are consistent with observations. The systems Ser X-1, GX 3+1, and 4U 1735-444 have accretion rates $l_{\mathrm{acc}} \approx 0.2$, 0.2 , and 0.25 (Kuulkers 2004), and they exhibit normal bursts with $\alpha \approx 5800,2100$, and 4400, respectively (in't Zand et al. 2003, 2004b). Figure 5 shows that delayed bursts occur at these accretion rates only if the CNO abundance in the accreted matter is high. Therefore, our theoretical models of normal bursts are consistent with the observed delayed bursts with $\alpha \gtrsim 1000$ only if the $\mathrm{CNO}$ abundance in the accreted matter in notably greater than solar.

The average decay times of normal bursts in all systems that exhibit superbursts are lower than the average decay times of normal bursts in systems that do not exhibit superbursts (in't Zand et al. 2003, 2004b). We plot the effective normal burst duration $t_{\mathrm{H}+\mathrm{He}}$ as a function of $l_{\mathrm{acc}}$ in Figure 7 , where $t_{\mathrm{H}+\mathrm{He}}$ equals the energy released via hydrogen and helium burning during a normal burst divided by the Eddington lu-

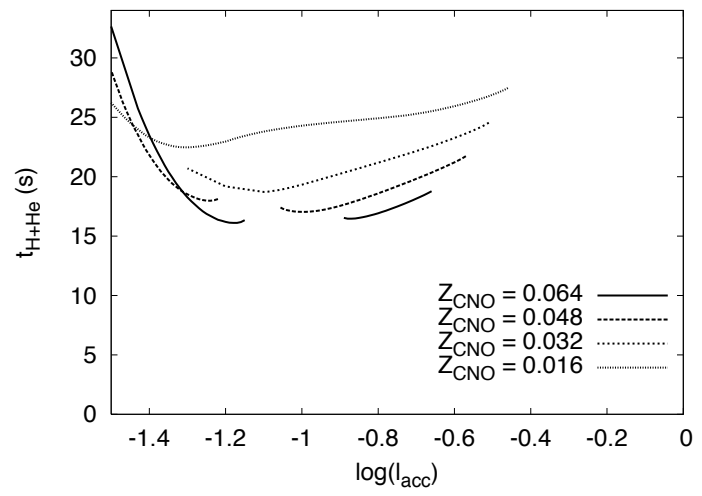

FIG. 7.- Effective normal burst duration as a function of accretion rate, calculated by dividing the total burst energy by the Eddington luminosity, for four different values of $Z_{\mathrm{CNO} \text { out }} . R=10.4 \mathrm{~km}, Q=5.2$ below the accreted layer, and the stellar core is assumed to emit neutrinos via mUrca reactions. Note that $t_{\mathrm{H}+\mathrm{He}}$ decreases with increasing $Z_{\mathrm{CNO} \text {,out }}$ for $l_{\mathrm{acc}} \gtrsim 0.06$.

minosity (see Narayan \& Heyl 2003). Figure 7 illustrates that systems with a large CNO abundance in the accreted matter will exhibit normal bursts with smaller durations. Again, our models of normal bursts are consistent with observations only if the CNO abundance in the accreted matter in high.

Several of the systems have $\alpha$-values that are even higher than those plotted in Figure 5. This could easily occur if the systems have accretion rates close to a critical accretion rate that separates stable burning and unstable burning (van Paradijs et al. 1979). The systems 4U 1636-536, KS 1731-260, Ser X-1, GX 3+1, and 4U 1735-444 all undergo episodes of irregular bursting behavior, which implies that they are accreting near such a critical rate (Lewin et al. 1987; Muno et al. 2000; Sztajno et al. 1983; den Hartog et al. 2003; van Paradijs et al. 1988b). Previous theoretical models predict that normal bursts will occur for all accretion rates below the critical rate above which helium burning is stable. Narayan \& Heyl (2003) find that the critical accretion rate is roughly $30 \%$ of the Eddington limit, which is consistent with observations (Cornelisse et al. 2003; Remillard et al. 2005b). Thus it is not surprising that Ser X-1, GX 3+1, and 4U 1735444 experience this irregular behavior, for their accretion rates are close to this upper bound. What is surprising is that $4 \mathrm{U}$ 1636-536 and KS 1731-260 experience irregular bursting behavior too, since $l_{\text {acc }} \approx 0.1$ for these two systems. This implies that the range of accretion rates at which normal bursts occur is not continuous, for there exists a range of accretion rates below $l_{\text {acc }} \approx 0.1$ in which normal bursts do not occur. The bursting behavior of KS 1731-260, which is a transient system, is particularly interesting. Muno et al. (2000) find that short, photospheric radius expansion bursts occur at high accretion rates, where $l_{\text {acc }} \sim 0.1$, and long bursts with no evidence of radius expansion occur at low accretion rates, where $l_{\text {acc }} \sim 0.01$, but no bursts occur at intermediate accretion rates. All of this is nicely reproduced in Figures 5 and 7 for models with CNO enhancement in the accreted matter. Shih et al. (2005) suggest that the accretion rate of 4U 1636-536 is currently decreasing with time. Our models predict that in this system in the near future either $\alpha$ will rise or normal bursts will cease altogether. This prediction should be testable with further observations.

The system GX $17+2$, which has an accretion rate $l_{\text {acc }} \approx 0.8$ and exhibits normal bursts with $\alpha \sim 1000$ as well as super- 
bursts, is still a mystery to us. Though there is little direct evidence that the accretion rate is so high, in't Zand et al. (2004a) conclude with reasonable certainty that the accretion luminosity is always greater than $60 \%$ of the Eddington limit. If this is indeed true, then clearly the observations of GX $17+2$ are inconsistent with our results. Theoretical models predict that systems that accrete at this rate will show superbursts given a sufficient amount of carbon (Cumming \& Bildsten 2001; Brown 2004; Cooper \& Narayan 2005). However, it is not understood why so much carbon should survive or why normal bursts should occur at such a high accretion rate. We offer two possible explanations, neither of which is without issues. First, we find that a reduction of the radiative opacities by roughly an order of magnitude will produce delayed mixed bursts at $l_{\text {acc }} \approx 0.8$ with $\alpha \sim 1000$ and substantially increase the carbon yield due to stable burning. GX 17+2 has a magnetic field that is much stronger than those of typical low mass X-ray binaries (Kuulkers et al. 2002a), and strong internal radial magnetic fields lower the radiative opacities of the ocean (Mészáros 1992; Lamb 2000). However, if an internal magnetic field were solely responsible for the opacity reduction, it would have to be $\sim 10^{13} \mathrm{G}$ (van Riper 1988), whereas Wijnands et al. (1996) derive an upper limit of $\approx 5 \times 10^{9} \mathrm{G}$ for this system. Therefore, we think this explanation is highly unlikely. Second, we find that lowering the hydrogen mass fraction of the accreted matter, $X_{\text {out }}$, by a factor of $\sim 2$ will produce delayed mixed bursts at $l_{\text {acc }} \approx 0.8$ with $\alpha \sim 1000$ and substantially increase the carbon yield due to stable burning. This will lower the burst durations as well. This explanation is definitely plausible, for theoretical evolutionary models of intermediate-mass X-ray binaries suggest that the secondaries will be hydrogen-poor (Podsiadlowski et al. 2002, 2004). Though some of the normal bursts observed in GX $17+2$ were quite short ( $\sim 10$ seconds), most of the observed normal bursts were rather long ( $\sim 10$ minutes), which implies that hydrogen was abundant in the ocean when the bursts occurred (Kahn \& Grindlay 1984; Tawara et al. 1984; Sztajno et al. 1986; Kuulkers et al. 1997, 2002a). This issue should be investigated further.

\section{OBSERVATIONAL EVIDENCE OF CNO ENHANCEMENT}

Evidence that the accreted material in compact binaries can have non-solar abundances is found in a variety of systems. For example, UV and X-ray spectroscopy of accreting white dwarfs has revealed anomalous $\mathrm{N} / \mathrm{C}$ emission line ratios in the accreted material in a number of systems (e.g. Gänsicke et al. 2003; Bonnet-Bidaud \& Mouchet 2004; Ramsay et al. 2005, and references therein). Since the abundances of the accreting material directly reflect the abundances of the mass donor star, various evolutionary scenarios have been proposed to explain these significant deviations from solar abundances. Given that hydrogen-rich donor stars will produce $\mathrm{CNO}$ deep within their cores, $\mathrm{CNO}$ processing as the mass donor star burns hydrogen along its main sequence will naturally produce $\mathrm{CNO}$ enriched gas, with most of the $\mathrm{C}$ converted into ${ }^{13} \mathrm{~N}$ during the $\mathrm{CNO}$ cycle due to the long $\beta$-decay timescale of ${ }^{13} \mathrm{~N}$. If these products reach the surface, we may expect to see significant non-solar abundances in the accretion flow. For example, Thorstensen et al. (2002) find an expected $\left(\mathrm{N} / \mathrm{N}_{\odot}\right) \approx 5-8$ if the mass donor star in the cataclysmic binary QZ Ser had undergone significant hydrogen burning before mass transfer onto the white dwarf had started. Alternatively, the mass donor may have lost (part of) its hydrogen envelope, exposing its $\mathrm{CNO}$ core and significantly boosting the $\mathrm{CNO}$ mass fraction of the accreting matter. Enhanced $\mathrm{CNO}$ abundances have also been reported for the black-hole X-ray binary XTE J1118 (Haswell et al. 2002), and Jimenez-Garate et al. (2005) report $4<(\mathrm{N} / \mathrm{O}) /(\mathrm{N} / \mathrm{O})_{\odot}<9$ in the high-mass $\mathrm{X}$-ray binary Her X-1. Other possible, though perhaps less likely, avenues by which the mass donor stars can become CNOenriched include carbon accretion onto the donor star during the asymptotic giant branch phase of the neutron star progenitor (de Kool \& Green 1995; Steinhardt \& Sasselov 2005) and ejecta capture from the supernova that begot the neutron star (e.g. Israelian et al. 1999). Not many abundance analyses for X-ray binaries have been published, but it appears that the accretion of CNO-enriched material occurs in a number of compact binaries harboring evolved mass donor stars. We note that that the soft X-ray transient $4 U$ 1608-522, in which Remillard et al. (2005a) observed a likely superburst, may contain an evolved secondary star (Wachter et al. 2002).

Unfortunately, abundance analyses for the systems that exhibit superbursts have not yet been reported. The large number of strong $\mathrm{C}, \mathrm{N}$, and $\mathrm{O}$ spectral lines in the UV regime make it the best window for such a study, but these analyses are severely impeded by interstellar extinction at those wavelengths. Strong $\mathrm{H}$ and $\mathrm{He}$ lines tend to dominate the optical spectroscopy, and the fact that these systems persistently accrete at $0.1 \lesssim l_{\text {acc }} \lesssim 0.25$ hampers the study of the photospheric composition of the donor star since the accretion light dominates. For such systems, the Bowen fluorescence blend near $\lambda 4640-4660 \AA$ has proven to be a useful indirect probe of the mass donor star (Steeghs \& Casares 2002). This blend consists of several N III components that are part of the Bowen fluorescence process, as well as nearby C III lines. Irradiation of the mass donor star leads to sharp and resolvable emission components throughout this blend originating from its surface. This was first demonstrated in Sco X-1, where the $\mathrm{N}$ and $\mathrm{C}$ components from the donor were detected at similar strengths (see Figure 1 in Steeghs \& Casares 2002). Hynes et al. (2004) report optical spectroscopy of the superburst source Ser X-1 and remark that only the N III lines were detected in the Bowen emission blend, and no $\mathrm{C}$ III emission was evident. Casares et al. (2004) present a compilation of Bowen blend profiles from a number of X-ray binaries including the superburst sources V801 Ara (also known as 4U 1636536) and V926 Sco (also known as 4U 1735-444). Again, in V801 Ara sharp components are detected from the N III components, but no sharp C III components are evident. For V926 $\mathrm{Sco}$, a spectral feature coinciding with one $\mathrm{C}$ III line is detected, but the second C III line is not detected making it less clear-cut for this source.

We remark that, since the N III transitions are part of the Bowen fluorescence process, whereas the C III are not, this blend cannot be used for quantitative abundance analysis. However, the dominance of $\mathrm{N}$ over $\mathrm{C}$ in two of the three observed superburst systems is certainly suggestive that the accreting matter may indeed be $\mathrm{CNO}$-enriched. A more detailed abundance analysis of these systems could test whether the composition of the accreting matter does in fact conform to the expectations of our model calculations.

\section{SUMMARY AND CONCLUSIONS}

By merging the theoretical superburst model of Cooper \& Narayan (2005) with the detailed nuclear reaction network of Mukhopadhyay \& Chakrabarti (2000), we have investigated the physical scenarios in which accreting neutron stars can both produce and preserve sufficient 
amounts of carbon fuel to trigger superbursts. We have constructed a total of 144 different models that span the possible ranges of neutron star thermal conductivities, core neutrino emission mechanisms, and areal radii, as well as the CNO abundances in the accreted material. We find that neutron stars that accrete hydrogen-rich material with $\mathrm{CNO}$ mass fractions less than or equal to that of the Sun will not exhibit superbursts, regardless of their accretion rates, conductivities, core neutrino emissivities, or radii. Neutron stars that accrete

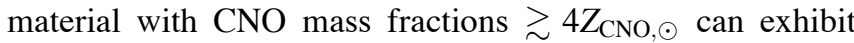
superbursts at accretion rates in the observed range, but only if the stellar radii are sufficiently large. We remark that the accreted carbon, nitrogen, and oxygen serve only as catalysts for hydrogen burning, and therefore only the sum of their individual mass fractions is important, not the individual mass fractions themselves.

Observationally, systems that exhibit superbursts differ from systems that do not exhibit superbursts not only in the amount of carbon that survives deep in the oceans of the neutron stars, but also in the nature of their normal bursts. Comparatively, the normal bursts observed in systems that exhibit superbursts are much shorter in duration, and they have $\alpha$ values that are much greater. Increasing the CNO abundance of the accreted material both decreases the durations of normal bursts and increases their $\alpha$-values, in agreement with observations. This is the first study in which the behavior of normal Type I X-ray bursts has been incorporated into models of systems that exhibit superbursts. Observers have discovered many compact stellar X-ray sources in which the accreted material is significantly non-solar. However, abundance analyses of the accreted material in systems that exhibit superbursts currently do not exist. We suggest that the secondaries of the low-mass X-ray binary systems Ser X-1, 4U 1254-690, 4U 1735-444, GX 3+1, KS 1731-260, and 4U 1636-536 are all rich in $\mathrm{CNO}$ elements. Although there is some indirect evi- dence in a few of these systems that the mass donor stars have indeed undergone $\mathrm{CNO}$-processing, more observations are required to either verify or refute this assertion.

One issue we have not addressed is the disparity between the superburst energies and recurrence times that have been observed and the energies and recurrence times derived from theoretical models. Specifically, all models predict that the energies and recurrence times should be roughly an order of magnitude larger than those observed. Theory and observations still disagree even if the thermal conductivity of the crust is low and the core temperature is high. Brown (2004) notes that observations of KS 1731-260 imply that the neutron star in this system in fact has a high thermal conductivity and a low core temperature (Wijnands et al. 2002), which illustrates the severity of this discrepancy. Furthermore, Cumming et al. (2005) show that neutrino emission via Cooper pairing of superfluid neutrons in the inner crust limits the temperature of the ocean to values well below the $\approx 6 \times 10^{8} \mathrm{~K}$ needed to trigger superbursts that match observations, and they suggest that an additional heating mechanism is required. Our models do not provide for such a mechanism, for we find that any additional heating due to the increased CNO abundance of the accreted material has a negligible effect on superburst energies and recurrence times. Superbursts may potentially be excellent probes into the interiors of neutron stars. Further progress in the physics of superbursts, combined with better observations, may ultimately lead to better understanding of not only neutron star interiors, but also fundamental physics.

We would like to thank Jorge Casares, Paul Green, Josh Grindlay, Hendrik Schatz, Jeffery McClintock, and Charles Steinhardt for helpful discussions, Jacob Fisker for kindly providing updated reaction rates, and the referee for useful comments and suggestions. D. S. acknowledges a Smithsonian Astrophysical Observatory Clay Fellowship. This work was supported in part by grant NNG04GL38G from NASA.

\section{REFERENCES}

Arnett, W. D. \& Truran, J. W. 1969, ApJ, 157, 339

Baym, G., Pethick, C., \& Pines, D. 1969, Nature, 224, 673

Bildsten, L. 1995, ApJ, 438, 852

Bildsten, L. 1998, in NATO ASIC Proc. 515: The Many Faces of Neutron Stars., 419

Bonnet-Bidaud, J.-M. \& Mouchet, M. 2004, in ASP Conf. Ser. 315: IAU Colloq. 190: Magnetic Cataclysmic Variables, 149

Brown, E. F. 2000, ApJ, 531, 988

-. 2004, ApJ, 614, L57

Casares, J., Steeghs, D., Hynes, R. I., Charles, P. A., Cornelisse, R., \& O'Brien, K. 2004, in Revista Mexicana de Astronomia y Astrofisica Conference Series, 21-22

Chakrabarti, S. K. \& Mukhopadhyay, B. 1999, A\&A, 344, 105

Clayton, D. D. 1983, Principles of Stellar Evolution and Nucleosynthesis (Chicago: Univ. Chicago Press)

Cooper, R. L. \& Narayan, R. 2004, AAS HEAD Meeting, 8, 17.28

-. 2005, ApJ, 629, 422

Cornelisse, R., in't Zand, J. J. M., Verbunt, F., Kuulkers, E., Heise, J., den Hartog, P. R., Cocchi, M., Natalucci, L., Bazzano, A., \& Ubertini, P. 2003, A\&A, 405, 1033

Cumming, A. 2003, ApJ, 595, 1077

-. 2004, Nuclear Physics B Proceedings Supplements, 132, 435

Cumming, A. \& Bildsten, L. 2001, ApJ, 559, L127

Cumming, A., Macbeth, J., in 't Zand, J. J. M., \& Page, D. 2005, submitted to ApJ (astro-ph/0508432)

de Kool, M. \& Green, P. J. 1995, ApJ, 449, 236

den Hartog, P. R., in't Zand, J. J. M., Kuulkers, E., Cornelisse, R., Heise, J., Bazzano, A., Cocchi, M., Natalucci, L., \& Ubertini, P. 2003, A\&A, 400, 633

Fisker, J. L., Brown, E. F., Liebendörfer, M., Thielemann, F.-K., \& Wiescher, M. 2005, Nuclear Physics A, 752, 604

Fisker, J. L., Gorres, J., Wiescher, M., \& Davids, B. 2004, preprint (astro-ph/0410561)
Fowler, W. A., Caughlan, G. R., \& Zimmerman, B. A. 1975, ARA\&A, 13 69

Fuller, G. M., Fowler, W. A., \& Newman, M. J. 1980, ApJS, 42, 447

-. 1982a, ApJ, 252, 715

$-.1982 \mathrm{~b}$, ApJS, 48, 279

Fushiki, I. \& Lamb, D. Q. 1987, ApJ, 317, 368

Gänsicke, B. T., Szkody, P., de Martino, D., Beuermann, K., Long, K. S., Sion, E. M., Knigge, C., Marsh, T., \& Hubeny, I. 2003, ApJ, 594, 443

Harris, M. J., Fowler, W. A., Caughlan, G. R., \& Zimmerman, B. A. 1983, ARA\&A, 21, 165

Haswell, C. A., Hynes, R. I., King, A. R., \& Schenker, K. 2002, MNRAS, 332,928

Hoyle, F. \& Fowler, W. A. 1965, in Quasi-Stellar Sources and Gravitational Collapse, 17

Hynes, R. I., Charles, P. A., van Zyl, L., Barnes, A., Steeghs, D., O’Brien, K., \& Casares, J. 2004, MNRAS, 348, 100

in't Zand, J. J. M., Cornelisse, R., \& Cumming, A. 2004a, A\&A, 426, 257

in't Zand, J. J. M., Cornelisse, R., Kuulkers, E., Heise, J., Verbunt, F., \& Cumming, A. 2004b, AAS HEAD Meeting, 8, 25.04

in't Zand, J. J. M., Kuulkers, E., Verbunt, F., Heise, J., \& Cornelisse, R. 2003 A\&A, 411, L487

Israelian, G., Rebolo, R., Basri, G., Casares, J., \& Martín, E. L. 1999, Nature, 401,142

Itoh, N. \& Kohyama, Y. 1993, ApJ, 404, 268

Jimenez-Garate, M. A., Raymond, J. C., Liedahl, D. A., \& Hailey, C. J. 2005, ApJ, 625, 931

Kahn, S. M. \& Grindlay, J. E. 1984, ApJ, 281, 826

Koike, O., Hashimoto, M., Kuromizu, R., \& Fujimoto, S. 2004, ApJ, 603, 242

Kuulkers, E. 2004, Nuclear Physics B Proceedings Supplements, 132, 466

-. 2005, The Astronomer's Telegram, 483, 1

Kuulkers, E., Homan, J., van der Klis, M., Lewin, W. H. G., \& Méndez, M. 2002a, A\&A, 382, 947 
Kuulkers, E., in't Zand, J., Homan, J., van Straaten, S., Altamirano, D., \& van der Klis, M. 2004, in AIP Conf. Proc. 714: X-ray Timing 2003: Rossi and Beyond, 257-260

Kuulkers, E., in't Zand, J. J. M., van Kerkwijk, M. H., Cornelisse, R., Smith, D. A., Heise, J., Bazzano, A., Cocchi, M., Natalucci, L., \& Ubertini, P. 2002b, A\&A, 382, 503

Kuulkers, E., van der Klis, M., Oosterbroek, T., van Paradijs, J., \& Lewin, W. H. G. 1997, MNRAS, 287, 495

Lamb, D. Q. 2000, ApJS, 127, 395

Lang, K. R. 1999, Astrophysical Formulae (New York: Springer)

Lewin, W. H. G., Penninx, W., van Paradijs, J., Damen, E., Sztajno, M. Truemper, J., \& van der Klis, M. 1987, ApJ, 319, 893

Mészáros, P. 1992, High-Energy Radiation from Magnetized Neutron Stars (Chicago: Univ. Chicago Press)

Mukhopadhyay, B. \& Chakrabarti, S. K. 2000, A\&A, 353, 1029

-.2001, ApJ, 555, 816

Muno, M. P., Fox, D. W., Morgan, E. H., \& Bildsten, L. 2000, ApJ, 542, 1016

Narayan, R. \& Heyl, J. S. 2003, ApJ, 599, 419

Podsiadlowski, P., Rappaport, S., \& Pfahl, E. 2004, in Revista Mexicana de Astronomia y Astrofisica Conference Series, 97-100

Podsiadlowski, P., Rappaport, S., \& Pfahl, E. D. 2002, ApJ, 565, 1107

Ramsay, G., Hakala, P., Marsh, T., Nelemans, G., Steeghs, D., \& Cropper, M. 2005, A\&A, 440, 675

Remillard, R., Morgan, E., \& The ASM Team at MIT, N. 2005a, The Astronomer's Telegram, 482, 1

Remillard, R. A., Lin, D., Cooper, R. L., \& Narayan, R. 2005b, submitted to ApJ (astro-ph/0509758)

Schatz, H., Aprahamian, A., Barnard, V., Bildsten, L., Cumming, A., Ouellette, M., Rauscher, T., Thielemann, F.-K., \& Wiescher, M. 2001, Physical Review Letters, 86, 3471

Schatz, H., Bildsten, L., Cumming, A., \& Ouellette, M. 2003, Nuclear Physics A, 718, 247

Schatz, H., Bildsten, L., Cumming, A., \& Wiescher, M. 1999, ApJ, 524, 1014

Shapiro, S. L. \& Teukolsky, S. A. 1983, Black Holes, White Dwarfs, and Neutron Stars: The Physics of Compact Objects (New York: Wiley)

Shih, I. C., Bird, A. J., Charles, P. A., Cornelisse, R., \& Tiramani, D. 2005, MNRAS, 593

Steeghs, D. \& Casares, J. 2002, ApJ, 568, 273

Steinhardt, C. L. \& Sasselov, D. D. 2005, preprint (astro-ph/0502152)
Strohmayer, T. \& Bildsten, L. 2005, in Compact Stellar X-Ray Sources, ed. W. H. G. Lewin and M. van der Klis (Cambridge: Cambridge Univ. Press), in press (astro-ph/0301544)

Strohmayer, T. E. \& Brown, E. F. 2002, ApJ, 566, 1045

Strohmayer, T. E. \& Markwardt, C. B. 2002, ApJ, 577, 337

Sztajno, M., Basinska, E. M., Cominsky, L. R., Marshall, F. J., \& Lewin, W. H. G. 1983, ApJ, 267, 713

Sztajno, M., van Paradijs, J., Lewin, W. H. G., Langmeier, A., Trumper, J., \& Pietsch, W. 1986, MNRAS, 222, 499

Tawara, Y., Hirano, T., Kii, T., Matsuoka, M., \& Murakami, T. 1984, PASJ, 36,861

Thielemann, F.-K. 1980, Ph.D. Thesis

Thorstensen, J. R., Fenton, W. H., Patterson, J., Kemp, J., Halpern, J., \& Baraffe, I. 2002, PASP, 114, 1117

Tillett, J. C. \& MacDonald, J. 1992, ApJ, 388, 555

van Paradijs, J., Cominsky, L., Lewin, W. H. G., \& Joss, P. C. 1979, Nature, 280,375

van Paradijs, J., Penninx, W., \& Lewin, W. H. G. 1988a, MNRAS, 233, 437

van Paradijs, J., Penninx, W., Lewin, W. H. G., Sztajno, M., \& Truemper, J. 1988b, A\&A, 192, 147

van Riper, K. A. 1988, ApJ, 329, 339

Wachter, S., Hoard, D. W., Bailyn, C. D., Corbel, S., \& Kaaret, P. 2002, ApJ, 568,901

Wagoner, R. V., Fowler, W. A., \& Hoyle, F. 1967, ApJ, 148, 3

Wallace, R. K. \& Woosley, S. E. 1981, ApJS, 45, 389

Wijnands, R. 2001, ApJ, 554, L59

Wijnands, R., Guainazzi, M., van der Klis, M., \& Méndez, M. 2002, ApJ, 573, L45

Wijnands, R. A. D., van der Klis, M., Psaltis, D., Lamb, F. K., Kuulkers, E., Dieters, S., van Paradijs, J., \& Lewin, W. H. G. 1996, ApJ, 469, L5+

Woosley, S. E., Arnett, W. D., \& Clayton, D. D. 1973, ApJS, 26, 231

Woosley, S. E., Heger, A., Cumming, A., Hoffman, R. D., Pruet, J., Rauscher, T., Fisker, J. L., Schatz, H., Brown, B. A., \& Wiescher, M. 2004, ApJS, 151,75

Yakovlev, D. G., Kaminker, A. D., Gnedin, O. Y., \& Haensel, P. 2001, Phys. Rep., 354, 1

Yakovlev, D. G., Levenfish, K. P., \& Shibanov, Y. A. 1999, Uspekhi Fizicheskikh Nauk, 42, 737

Yakovlev, D. G. \& Pethick, C. J. 2004, ARA\&A, 42, 169 